\title{
Review of clinical trial settings of drugs commonly used in primary care and approved between 2005 and 2012
}

\author{
Jason R. Crossley, $B A^{7}$, Tina P. Tan, $B A^{2}$, Keisha Herbin Smith, $M A^{2}$, Joseph S. Ross, MD, $M H S^{3,4,5}$, \\ and Daniel J. Merenstein, $M D^{2}$
}

'Georgetown University School of Medicine, Washington, DC, USA; ${ }^{2}$ Department of Family Medicine, Georgetown University Medical Center, Washington, DC, USA; ${ }^{3}$ The Center for Outcomes Research and Evaluation, Yale-New Haven Hospital, New Haven, CT, USA; ${ }^{4}$ Section of General Internal Medicine and the National Clinician Scholars Program, Department of Medicine, Yale School of Medicine, New Haven, CT, USA;

${ }^{5}$ Department of Health Policy and Management, Yale School of Public Health, New Haven, CT, USA.

KEY WORDS: FDA; Primary care; Pivotal studies.

J Gen Intern Med 33(9):1431-2

DOI: $10.1007 / \mathrm{s} 11606-018-4486-7$

(c) Society of General Internal Medicine 2018

\section{INTRODUCTION}

The approval of new drugs by the US Food and Drug Administration (FDA) depends on demonstration of their safety and efficacy through clinical studies. To best inform clinical practice, these pivotal trials would ideally be conducted in care settings where the drugs will ultimately be used. For example, drugs used to treat diabetes mellitus are most frequently prescribed to patients by primary care physicians in office-based settings, yet trials used to support their approval may be conducted among patients receiving care from endocrinologists, in acute care settings and/or in non-US-based healthcare delivery systems ${ }^{1}$. Our objective was to characterize the patient populations and settings of pivotal trials supporting FDA approvals from 2005 to 2012 for new drugs used for indications most commonly treated in the primary care setting.

\section{METHODS}

Using data previously collected for all new drugs approved by the FDA between 2005 and $2012^{2}$, we identified drugs originally approved for use for indications most commonly treated in primary care, along with the pivotal trials that supported their approvals. Using publicly available FDA documents, including medical officer reviews, and publications ${ }^{3}$, four independent reviewers collected the following characteristics for each trial: recruitment setting, principal investigator (PI) specialty, and study country. Discrepancies were resolved by discussion. We used descriptive statistics to quantify these trial characteristics.

\section{RESULTS}

Forty new drugs approved between 2005 and 2012 were determined as being most commonly used in primary care

Published online May 18, 2018 settings (Table 1). These drugs were approved on the basis of 151 pivotal trials, for which 129 corresponding publications were identified. Of these publications, we were unable to ascertain the trial setting for $96(74 \%), 22(17 \%)$ were conducted in non-primary care settings, $11(8 \%)$ in both primary care and non-primary care settings, and none exclusively in primary care settings (Table 2). PI specialty could be determined for 121 (89.4\%), of which 116 (95.9\%) were specialty physicians and five were primary care physicians (internal medicine, 4; family medicine, 1). Among the 129 publications, $38(30 \%)$ were conducted exclusively in the USA, 24 (19\%) exclusively outside the USA (19\%), 39 (31\%) both inside and outside the USA, and 27 (21\%) had no discernible study locale.

\section{DISCUSSION}

Among the new drugs approved between 2005 and 2012 that are most commonly used in the primary care setting, threequarters of pivotal trials supporting FDA approval did not provide sufficient information in publicly available FDA documents and corresponding publications to determine if they were conducted in primary care settings. Based on the information available, we determined that $8 \%$ of trials were partially conducted in primary care settings - none exclusively so. Our results suggest that the approval of these new drugs were predominantly supported by evidence generated in subspecialty settings, despite that most end users of these therapies will likely be patients and prescribers in primary care settings.

Evaluating drugs in settings that do not resemble where they will most often be used may result in outsized estimates of benefit — because of the risk-treatment paradox ${ }^{4}$-as well as safety risk, since patients with more comorbidities who are taking more medications - and thus more likely to require specialty care-are more likely to experience adverse drug events ${ }^{5}$. The Randomized Aldactone Evaluation Study was one instance; following publication, the rate of hospitalization for hyperkalemia increased from 2.4 to 11.0 per 1000 patients $(P<0.001)$, and the associated mortality increased from 0.3 to 2.0 per 1000 patients $(P<0.001)$ from 1994 to 2001 , 
Table 1 Characteristics of new drugs for indications most commonly treated in primary care approved by the FDA from 2005 to $2012(n=40)$.

\begin{tabular}{lll}
\hline \hline & $N$ & $\%$ \\
\hline Agent type & & \\
Small molecule & 38 & 95 \\
Biologic & 2 & 5 \\
Approval year & & \\
2005 & 6 & 15 \\
2006 & 7 & 17.5 \\
2007 & 2 & 5 \\
2008 & 4 & 10 \\
2009 & 7 & 17.5 \\
2010 & 7 & 17.5 \\
2011 & 7 & 17.5 \\
Therapeutic area & & \\
Autoimmune and musculoskeletal & 2 & 5 \\
Cardiovascular disease, diabetes mellitus and & 13 & 32.5 \\
hyperlipidemia & & \\
Dermatology & 4 & 10 \\
Infectious disease & 4 & 10 \\
Neurology & 1 & 2.5 \\
Psychiatry & 4 & 10 \\
Other & 12 & 30 \\
\hline
\end{tabular}

presumably due to the different patient populations seen in trial and practice settings ${ }^{6}$. There are other important differences in care processes between primary care and specialty care settings that may affect medication management, including visit length, ancillary staff availability, and educational support.

Our estimates are deliberately conservative, as the descriptive text provided in FDA documents and publications was often vague. It is possible that settings described ambiguously as "clinics" were in fact primary care clinics, but without explicit detail; we categorized these as indeterminate. This information could be clarified through the FDA's Drug Trials Snapshots initiative (www.fda.gov/ Drugs/InformationOnDrugs/ucm412998.htm), which provides demographic characteristics of trial participants of newly approved drugs, offering additional regulatory transparency and information to patients and clinicians. Future efforts should encourage trial recruitment in clinical settings in which the drugs will most commonly be used, ensuring new drug evaluations that can best inform patient care.

Table 2 Patient recruitment setting and country for pivotal trials of new drugs for indications most commonly treated in primary care supporting the approval by the FDA from 2005 to $2012(n=129)$.

\begin{tabular}{lll}
\hline \hline & Count & $\%$ \\
\hline & & \\
Study setting & & 0 \\
Primary care & 0 & 17.1 \\
Non-primary care & 22 & 8.5 \\
Both primary care and non-primary care & 11 & 74.4 \\
Could not be determined & 96 & 29.5 \\
Study country & & 18.6 \\
The USA & 38 & 30.2 \\
Outside of the USA & 24 & 21.7 \\
Both the USA and outside of the USA & 39 & \\
Could not be determined & 28 & \\
\hline
\end{tabular}

Corresponding Author: Daniel J. Merenstein, MD; Department of Family Medicine Georgetown University Medical Center, Washington, DC, USA (e-mail: djm23@georgetown.edu).

\section{Compliance with ethical standards:}

Conflict of Interest: DM has consulted for Pharmavite, Reckitt Benckiser and Debevoise \& Plimpton this past year. The Dannon Company, Lifeway Foods, Inc., Nestlé, and Cargill, Inc., have previously provided funding for separate clinical trials through Georgetown University. In the past 36 months, Dr. Ross has received research support through Yale University from Johnson and Johnson to develop methods of clinical trial data sharing, from Medtronic, Inc., and the Food and Drug Administration (FDA) to develop methods for post-market surveillance of medical devices (UO1FDO04585), from the Food and Drug Administration to establish Yale-Mayo Clinic Center for Excellence in Regulatory Science and Innovation (CERSI) program (UO1FDO05938), from the Blue Cross Blue Shield Association to better understand medical technology evaluation, from the Centers of Medicare and Medicaid Services (CMS) to develop and maintain performance measures that are used for public reporting, from the Agency for Healthcare Research and Quality to examine community predictors of healthcare quality (RO1HSO22882), and from the Laura and John Arnold Foundation to establish the Good Pharma Scorecard at Bioethics International and to establish the Collaboration for Research Integrity and Transparency (CRIT) at Yale. The other authors report no potential conflicts of interest.

\section{REFERENCES}

1. Leinung MC, Gianoukakis AG, Lee DW, Jeronis SL, Desemone J. Comparison of diabetes care provided by an endocrinology clinic and a primary-care clinic. Endocrine practice: official journal of the American College of Endocrinology and the American Association of Clinical Endocrinologists. 2000;6(5):361-366. http://www.ncbi.nlm.nih.gov/pubmed/ 11141586. doi: https://doi.org/10.4158/EP.6.5.361.

2. Downing NS, Aminawung JA, Shah ND, Krumholz HM, Ross JS. Clinical trial evidence supporting FDA approval of novel therapeutic agents, 20052012. JAMA. 2014;311(4):368-377. https://doi.org/10.1001/jama.2013. 282034.

3. Smithy JW, Downing NS, Ross JS. Publication of Pivotal Efficacy Trials for Novel Therapeutic Agents Approved Between 2005 and 2011: A Crosssectional Study. JAMA Intern Med. 2014;174(9):1518-1520. https://doi. org/10.1001/jamainternmed.2014.3438

4. Ko DT, Mamdani M, Alter DA. Lipid-Lowering Therapy With Statins in High-Risk Elderly Patients: The Treatment-Risk Paradox. JAMA. 2004;291(15):1864-1870. https://doi.org/10.1001/jama.291.15.1864.

5. Field TS, Gurwitz JH, Avorn J, et al. Risk Factors for Adverse Drug Events Among Nursing Home Residents. Arch Intern Med. 2001;161(13):16291634. doi: https://doi.org/10.1001/archinte.161.13.1629.

6. Juurlink DN, Mamdani MM, Lee DS, et al. Rates of hyperkalemia after publication of the Randomized Aldactone Evaluation Study. N Engl J Med. 2004 Aug 5;351(6):543-551. https://doi.org/10.1056/NEJMoa040135 